\title{
Telegraph noise in Markovian master equation for electron transport through molecular junctions
}

Daniel S. Kosov

Citation: The Journal of Chemical Physics 148, 184108 (2018); doi: 10.1063/1.5033354

View online: https://doi.org/10.1063/1.5033354

View Table of Contents: http://aip.scitation.org/toc/jcp/148/18

Published by the American Institute of Physics

\section{Articles you may be interested in}

Perspective: Theory of quantum transport in molecular junctions

The Journal of Chemical Physics 148, 030901 (2018); 10.1063/1.5003306

Communication: Symmetrical quasi-classical analysis of linear optical spectroscopy

The Journal of Chemical Physics 148, 181102 (2018); 10.1063/1.5031788

Emergence of Landauer transport from quantum dynamics: A model Hamiltonian approach

The Journal of Chemical Physics 148, 144707 (2018); 10.1063/1.5009815

Announcement: Top reviewers for The Journal of Chemical Physics 2017

The Journal of Chemical Physics 149, 010201 (2018); 10.1063/1.5043197

Fast-forward Langevin dynamics with momentum flips

The Journal of Chemical Physics 148, 184109 (2018); 10.1063/1.5029833

Two-dimensional Fano lineshapes: Excited-state absorption contributions

The Journal of Chemical Physics 148, 184201 (2018); 10.1063/1.5019376

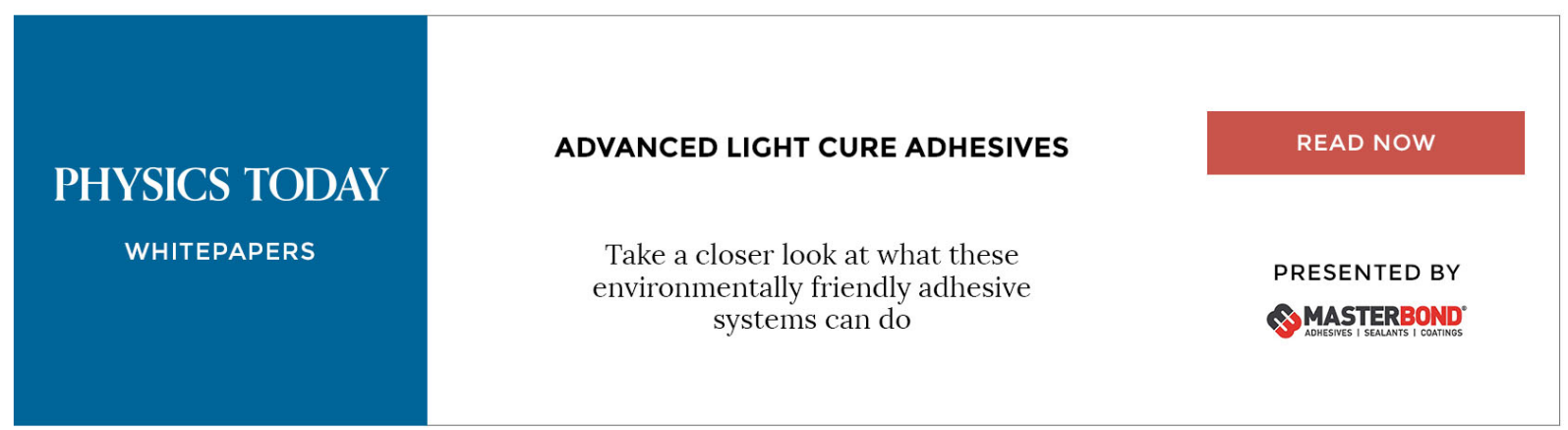




\title{
Telegraph noise in Markovian master equation for electron transport through molecular junctions
}

\author{
Daniel S. Kosov \\ College of Science and Engineering, James Cook University, Townsville, QLD 4811, Australia
}

(Received 5 April 2018; accepted 27 April 2018; published online 10 May 2018)

\begin{abstract}
We present a theoretical approach to solve the Markovian master equation for quantum transport with stochastic telegraph noise. Considering probabilities as functionals of a random telegraph process, we use Novikov's functional method to convert the stochastic master equation to a set of deterministic differential equations. The equations are then solved in the Laplace space, and the expression for the probability vector averaged over the ensemble of realisations of the stochastic process is obtained. We apply the theory to study the manifestations of telegraph noise in the transport properties of molecular junctions. We consider the quantum electron transport in a resonant-level molecule as well as polaronic regime transport in a molecular junction with electron-vibration interaction. Published by AIP Publishing. https://doi.org/10.1063/1.5033354
\end{abstract}

\section{INTRODUCTION}

Recently, there have been significant experimental and theoretical interest in studying the fluctuations of the electric properties of molecular junctions. ${ }^{1-16}$ There are two distinctly different types of stochastic fluctuations in current-carrying molecular junctions. On the fundamental level, there is always noise due to the probabilistic nature of quantum mechanics. ${ }^{17}$ Moreover, the electron transport in molecular junctions often demonstrates random switching of the electric current between multiple different values due to the presence of localised electronic states, various geometric stochastic conformational switching, and current-triggered irregular motion of interface atoms. ${ }^{18-22}$

The scope of theoretical work on telegraph noise in steady state quantum transport is still very limited..$^{23-26}$ The resonant electron tunneling through a "two-level fluctuator" was studied more than 20 years ago using Green's function methods. ${ }^{23,24}$ Then, only recently, Gurvitz et al. described both time-dependent and steady state electric current through the single-electron transistor with random Markovian jumps of the single-electron energy level. ${ }^{25}$ The approach, which incorporates telegraph noise into Keldysh nonequilibrium Green's functions, was proposed last year to study quantum heat transport through a single fluctuating level electronic junction. ${ }^{26}$ In this paper, we develop a statistical theory of electron transport in molecular junctions with time-varying parameters, which can switch stochastically between two different values (telegraphic noise). We work with the Markovian master equation and we incorporate the telegraph process into electron transfer rates-it results in the stochastic master equation. The stochastic differential equations, which represent the master equation with telegraph noise, are converted to the closed system of deterministic differential equations by using Novikov's method of stochastic functionals. ${ }^{27}$ The method was first proposed by Novikov in 1964 in the theory of turbulence ${ }^{27}$ and then developed in detail by
Klyatskin in the series of monographs. ${ }^{28-30}$ A similar approach was recently employed to study the electron transport through a single fluctuating energy level. ${ }^{25}$ Telegraph stochastic processes considered in this paper are closely related to Markovian kinetic equations with the KeilsonStorer kernel, ${ }^{31}$ which have many applications in chemical kinetics. ${ }^{32-37}$

This paper is organised as follows. Section II describes the solution of the general Markovian master equation with rates, which depend on a stochastic telegraph process. In Sec. III, we apply the theory to the problem of electron transport through a molecular junction: first, we consider a simple case of a single resonant molecular orbital and, second, we discuss the telegraph noise in a molecular junction with electron-vibrational interaction. Section IV summarises the main results of the paper.

We use natural units for quantum transport throughout the paper: $\hbar=k_{B}=e=1$.

\section{MARKOVIAN MASTER EQUATION WITH TELEGRAPH NOISE}

In this section, we consider a general problem of finding the solution for the master equation with time dependent rates which undergo jumps between two different values at specific but ransom times (telegraph noise). We discuss general theory first (applicable to any Markovian dynamics with telegraph noise) and then apply the theory to quantum electron transport through molecular junction in Sec. III. We begin with the Markovian master equation

$$
P_{k}(t)=\sum_{k^{\prime}} W_{k k^{\prime}} P_{k^{\prime}}(t)
$$

where $P_{k}(t)$ is the probability of observing our system in state $k$ at time $t$ and $W_{k k^{\prime}}$ is the transition probability per unit time (rate) from state $k^{\prime}$ to state $k$. In matrix form, master equation (1) can be compactly written as 


$$
\frac{d \mathbf{P}(t)}{d t}=\mathbf{W P}(t) .
$$

Suppose that the parameters of the system experience some stochastic variations, which result in the telegraph noise in the rates of the master equation. The master equation becomes

$$
\frac{d \mathbf{P}(t)}{d t}=\mathbf{W P}(t)+\xi(t) \mathbf{A P}(t) .
$$

Here function $\xi(t)$ describes the stochastic telegraph noise process, the function switches stochastically between two values as time progresses

$$
\xi(t)=\gamma(-1)^{n(0, t)},
$$

where $n\left(t, t^{\prime}\right)$ is the random sequence of integer numbers describing the number of telegraph jumps within the time interval $\left[t, t^{\prime}\right] .{ }^{30}$ It means that the dynamics of the probabilities is governed by the time-dependent matrix that switches stochastically between $\mathbf{W}+\mathbf{A}$ and $\mathbf{W}-\mathbf{A}$, and the probability vector itself $\mathbf{P}(t)$ becomes a stochastic function of time. The matrix $\mathbf{A}$ is not completely arbitrary, it should have two general properties of $\mathbf{W}$-matrices of a Markovian master equation ${ }^{38}$

$$
W_{k k^{\prime}} \pm \gamma A_{k k^{\prime}} \geq 0 \text { for } k \neq k^{\prime}
$$

to preserve the positivity of the probabilities and

$$
\sum_{k} A_{k k^{\prime}}=0 \text { for any } k^{\prime}
$$

to maintain the proper normalization of the probability vector at each moment of time despite of the stochastic discontinuous changes of the rate matrix.

We assume that the distribution of times at which telegraph jumps to occur is the Poisson point distribution. It means that the probability to observe $n$ jumps during the time interval $\left[t, t^{\prime}\right]$ is

$$
p_{n\left(t, t^{\prime}\right)=n}=\frac{\left(\alpha\left(t-t^{\prime}\right)\right)^{n}}{n !} e^{-\alpha\left(t-t^{\prime}\right)}
$$

with an average number of telegraph jumps during the time interval

$$
\overline{n\left(t, t^{\prime}\right)}=\sum_{n=0}^{\infty} n p_{n\left(t, t^{\prime}\right)=n}=\alpha\left(t-t^{\prime}\right) .
$$

Using the Poisson point distribution, we compute the first few moments of the stochastic function $\xi(t)$ averaging over the realisations of the stochastic process. The average value of the stochastic process $\xi(t)$ at time $\mathrm{t}$ is

$$
\langle\xi(t)\rangle_{\xi}=\sum_{n=0}^{\infty} p_{n(0, t)=n} \gamma(-1)^{n}=\gamma e^{-2 \alpha t},
$$

where $\langle\ldots\rangle_{\xi}$ means the averaging over all possible realization of stochastic process $\xi(t)$. Two-time correlation function for $t_{1}>t_{2}$ is

$$
\begin{aligned}
\left\langle\xi\left(t_{1}\right) \xi\left(t_{2}\right)\right\rangle_{\xi} & =\left\langle\gamma(-1)^{n\left(0, t_{1}\right)} \gamma(-1)^{n\left(0, t_{2}\right)}\right\rangle_{\xi} \\
& =\gamma^{2}\left\langle(-1)^{n\left(t_{2}, t_{1}\right)}\right\rangle_{\xi}=\gamma^{2} e^{-2 \alpha\left(t_{1}-t_{2}\right)}
\end{aligned}
$$

and all higher order correlation functions can be factorised in terms of average process and two-time correlation functions. ${ }^{30}$

Let us now average the stochastic master equation (3) over the ensemble of telegraphic noise realisations

$$
\frac{d}{d t}\langle\mathbf{P}(t)\rangle_{\xi}=\mathbf{W}\langle\mathbf{P}(t)\rangle_{\xi}+\mathbf{A}\langle\xi(t) \mathbf{P}(t)\rangle_{\xi}
$$

Differential equation (11) is not yet in a complete form since we do not know how to compute $\langle\xi(t) \mathbf{P}(t)\rangle_{\xi}$.

To evaluate $\langle\xi(t) \mathbf{P}(t)\rangle_{\xi}$, we notice that $\mathbf{P}(t)$ can be considered as a functional of the stochastic process $\xi(t)$

$$
\mathbf{P}(t)=\mathbf{P}[\xi](t) .
$$

Next we use Novikov's method of computing averages of products of functional of the stochastic process with the process function itself. ${ }^{27-30}$ Following the Novikov ideas, ${ }^{27}$ we introduce

$$
\langle\xi(t) \mathbf{P}[\xi+z](t)\rangle_{\xi},
$$

where $z(t)$ is an arbitrary reasonably smooth deterministic function. We expand $\mathbf{P}[\xi+z](t)$ in the Taylor-like series using functional differentiation around $\xi(t)=0$

$$
\mathbf{P}[\xi+z](t)=\exp \left\{\int_{0}^{\infty} d t_{1} \xi\left(t_{1}\right) \frac{\delta}{\delta z\left(t_{1}\right)}\right\} \mathbf{P}[z](t) .
$$

We notice that $\mathbf{P}[z](t)$ is a completely deterministic functional and all stochastic variables are now localised in the operator exponent in (14); therefore

$$
\langle\xi(t) \mathbf{P}[\xi+z](t)\rangle_{\xi}=\left\langle\xi(t) \exp \left\{\int_{0}^{\infty} d t_{1} \xi\left(t_{1}\right) \frac{\delta}{\delta z\left(t_{1}\right)}\right\}\right\rangle_{\xi} \mathbf{P}[z](t) .
$$

Expanding the operator exponent in (15), we get

$$
\begin{aligned}
\langle\xi(t) \mathbf{P}[\xi+z](t)\rangle_{\xi} & =\left\langle\xi(t)\left[1+\sum_{k=1}^{\infty} \frac{1}{k !} \int_{0}^{\infty} d t_{1} \int_{0}^{\infty} d t_{2} \ldots \int_{0}^{\infty} d t_{k} \xi\left(t_{1}\right) \xi\left(t_{2}\right) \ldots \xi\left(t_{k}\right) \frac{\delta}{\delta z\left(t_{1}\right)} \frac{\delta}{\delta z\left(t_{2}\right)} \ldots \frac{\delta}{\delta z\left(t_{k}\right)}\right]\right\rangle_{\xi} \mathbf{P}[z](t) \\
& =\left[\langle\xi(t)\rangle_{\xi}+\sum_{k=1}^{\infty} \int_{0}^{\infty} d t_{1} \int_{0}^{t_{1}} d t_{2} \ldots \int_{0}^{t_{k-1}} d t_{k}\left\langle\xi(t) \xi\left(t_{1}\right)\right\rangle_{\xi}\left\langle\xi\left(t_{2}\right) \ldots \xi\left(t_{k}\right)\right\rangle_{\xi} \frac{\delta}{\delta z\left(t_{1}\right)} \frac{\delta}{\delta z\left(t_{2}\right)} \ldots \frac{\delta}{\delta z\left(t_{k}\right)}\right] \mathbf{P}[z](t) \\
& =\left[\langle\xi(t)\rangle_{\xi}+\int_{0}^{\infty} d t_{1}\left\langle\xi(t) \xi\left(t_{1}\right)\right\rangle_{\xi} \frac{\delta}{\delta z\left(t_{1}\right)}\left\langle\exp \left\{\int_{0}^{t_{1}} d t^{\prime} \xi\left(t^{\prime}\right) \frac{\delta}{\delta z\left(t^{\prime}\right)}\right\}\right\rangle_{\xi}\right] \mathbf{P}[z](t) .
\end{aligned}
$$

Substituting expressions for the moments of the stochastic process (9) and (10) yields 


$$
\langle\xi(t) \mathbf{P}[\xi+z](t)\rangle_{\xi}=\left[\gamma e^{-2 \alpha t}+\gamma^{2} \int_{0}^{\infty} d t_{1} e^{-2 \alpha\left(t-t_{1}\right)} \frac{\delta}{\delta z\left(t_{1}\right)}\left\langle\exp \left\{\int_{0}^{t_{1}} d t^{\prime} \xi\left(t^{\prime}\right) \frac{\delta}{\delta z\left(t^{\prime}\right)}\right\}\right\rangle_{\xi}\right] \mathbf{P}[z](t) .
$$

We differentiate the lhs and the rhs of this equation with respect to $t$ and obtain

$$
\left(\frac{d}{d t}+2 \alpha\right)\langle\xi(t) \mathbf{P}[\xi+z](t)\rangle_{\xi}=\left[\gamma e^{-2 \alpha t}+\gamma^{2} \int_{0}^{\infty} d t_{1} e^{-2 \alpha\left(t-t_{1}\right)} \frac{\delta}{\delta z\left(t_{1}\right)}\left\langle\exp \left\{\int_{0}^{t_{1}} d t^{\prime} \xi\left(t^{\prime}\right) \frac{\delta}{\delta z\left(t^{\prime}\right)}\right\}\right\rangle_{\xi}\right] \frac{d}{d t} \mathbf{P}[z](t),
$$

which is simply

$$
\left(\frac{d}{d t}+2 \alpha\right)\langle\xi(t) \mathbf{P}[\xi+z](t)\rangle_{\xi}=\left\langle\xi(t) \frac{d}{d t} \mathbf{P}[\xi+z](t)\right\rangle_{\xi} .
$$

Setting $z(t)=0$ in the above equation, we get $^{30,39}$

$$
\left(\frac{d}{d t}+2 \alpha\right)\langle\xi(t) \mathbf{P}[\xi](t)\rangle_{\xi}=\left\langle\xi(t) \frac{d}{d t} \mathbf{P}[\xi](t)\right\rangle_{\xi} .
$$

Equation (20) is sometimes called the Shapiro-Loginov differential formula. ${ }^{25,39}$ Substituting $\frac{d}{d t} \mathbf{P}[\xi](t)$ from the initial master equation (3) into the right-hand side of (20) gives

$$
\left(\frac{d}{d t}+2 \alpha-\mathbf{W}\right)\langle\xi(t) \mathbf{P}(t)\rangle_{\xi}=\gamma^{2} \mathbf{A}\langle\mathbf{P}(t)\rangle_{\xi} .
$$

We omit here and throughout the rest of the paper the functional dependence notation in $\mathbf{P}$ for brevity. Note that although (20) can be generalized to an arbitrary Gauss-Markov stochastic process ${ }^{39}$ Eq. (21) already requires that $\xi(t)$ should be a dichotomic process in order to close the hierarchy of the differential equations at the $\langle\xi \mathbf{P}\rangle_{\xi}$ term.

We have converted the initial stochastic master equation (3) to the closed system of two coupled deterministic differential equations (11) and (21). Next we introduce function

$$
\mathbf{F}(t)=\langle\xi(t) \mathbf{P}(t)\rangle_{\xi}
$$

and perform the Laplace transformation on the system of differential equations (11) and (21). We find

$$
\begin{gathered}
s\langle\widetilde{\mathbf{P}}(s)\rangle_{\xi}-\mathbf{P}(0)=\mathbf{W}\langle\widetilde{\mathbf{P}}(s)\rangle_{\xi}+\mathbf{A} \widetilde{\mathbf{F}}(s), \\
(s \mathbf{I}+2 \alpha-\mathbf{W}) \widetilde{\mathbf{F}}(s)-\gamma \mathbf{P}(0)=\gamma^{2} \mathbf{A}\langle\widetilde{\mathbf{P}}(s)\rangle_{\xi},
\end{gathered}
$$

where "tilde" denotes functions in the Laplace space, for example, $\widetilde{\mathbf{P}}(s)=\int_{0}^{\infty} d t e^{-s t} \mathbf{P}(t)$ and $\mathbf{I}$ is the identity matrix. While deriving [(23) and (24)], we took into account that the probability vector $\mathbf{P}(t)$ does not depend on stochastic variable $\xi$ at initial time $t=0$, therefore $\langle\mathbf{P}(0)\rangle_{\xi}=\mathbf{P}(0)$ and

$$
\langle\xi(0) \mathbf{P}(0)\rangle_{\xi}=\langle\xi(0)\rangle_{\xi} \mathbf{P}(0)=\gamma \mathbf{P}(0) .
$$

Eliminating $\widetilde{\mathbf{F}}(s)$ from (23) and (24) gives the following expression for the noise averaged probability vector in Laplace space

$$
\begin{aligned}
\langle\widetilde{\mathbf{P}}(s)\rangle_{\xi}= & {\left[(s \mathbf{I}-\mathbf{W})-\gamma^{2} \mathbf{A} \frac{1}{(s+2 \alpha) \mathbf{I}-\mathbf{W}} \mathbf{A}\right]^{-1} } \\
& \times\left[\mathbf{I}+\gamma \mathbf{A} \frac{1}{(s+2 \alpha) \mathbf{I}-\mathbf{W}}\right] \mathbf{P}(0) .
\end{aligned}
$$

\section{APPLICATIONS TO THE QUANTUM TRANSPORT}

\section{A. Electron transport through single-resonant level with telegraph noise in the contacts}

Let us consider the electron transport through a molecular junction represented by a single resonant molecular orbital connected to two leads (left and right) held at different chemical potentials. The electron spin is not considered; therefore, the molecular orbital can only contain zero or one electron. The Markovian master equation is given by (3), where matrix $\mathbf{W}$ is

$$
\mathbf{W}=\left[\begin{array}{cc}
-T_{10} & T_{01} \\
T_{10} & -T_{01}
\end{array}\right]
$$

and $\mathbf{P}(t)$ is the probability vector

$$
\mathbf{P}(t)=\left[\begin{array}{l}
P_{0}(t) \\
P_{1}(t)
\end{array}\right] .
$$

Here $P_{0}(t)$ is the probability that the molecular orbital has zero electrons at time $t$ and $P_{1}(t)$ is the probability for the orbital to be occupied by one electron at time $t$. The dynamical evolution of these probabilities is determined by two rates: $T_{01}$ describes the transition from the occupied to empty state and $T_{10}$ is the rate for the opposite process. The total rates consist of two contributions from left and right electrodes

$$
T_{10}=T_{10}^{L}+T_{10}^{R}, \quad T_{01}=T_{01}^{L}+T_{01}^{R} .
$$

The partial rates are given by the standard Fermi golden rule expressions

$$
\begin{aligned}
& T_{01}^{L}=\Gamma_{L} f_{L}, \quad T_{01}^{R}=\Gamma_{R} f_{R}, \\
& T_{10}^{L}=\Gamma_{L}\left(1-f_{L}\right), \quad T_{10}^{R}=\Gamma_{R}\left(1-f_{R}\right),
\end{aligned}
$$

where $\Gamma_{L / R}$ are molecular level energy broadening functions due to the coupling of the left/right electrode. Fermi occupation numbers for left and right electrodes are

$$
f_{L}=\left[1+e^{\left(\epsilon-\mu_{L}\right) / T_{L}}\right]^{-1}, \quad f_{R}=\left[1+e^{\left(\epsilon-\mu_{R}\right) / T_{R}}\right]^{-1} .
$$

Voltage bias is defined as the difference between left and right electrodes' chemical potentials: $V=\mu_{L}-\mu_{R}$.

Let us introduce the telegraph noise into the master equation. Recently Gurvitz et al. ${ }^{25}$ used a similar method to model a single electron transistor with an energy level which can stochastically jump between two given values. The most prevailing scenario for molecular junctions is not the fluctuation of the molecular orbital itself but rather the telegraph noise originating from various processes on the moleculemetal interfaces. ${ }^{18}$ To model this setting, we assume that the coupling between the molecule and the left electrode 
$\Gamma_{L}$ undergoes telegraph type stochastic oscillations (the choice of left electrode is arbitrary, we can also consider the coupling between molecule and the right electrode $\Gamma_{R}$ )

$$
\Gamma_{L}(t)=\Gamma_{L}(1+\xi(t)) .
$$

Here $\xi(t)$ is a stochastic telegraph noise variable, which can switch between two states. Therefore, matrix $\mathbf{A}$ is

$$
\mathbf{A}=\left[\begin{array}{cc}
-T_{10}^{L} & T_{01}^{L} \\
T_{10}^{L} & -T_{01}^{L}
\end{array}\right]
$$

With this definition of matrix $\mathbf{A}$, the amplitude of the stochastic telegraph process $|\gamma|$ should be $\leq 1$ to preserve the positivity of the probabilities (5).

The $\operatorname{limit}_{\lim } \rightarrow 0 \tilde{s}(s)$ of the Laplace transformed function corresponds to the asymptotic time limit of the real time function $\lim _{t \rightarrow \infty} f(t)$. Therefore, the stationary (nonequilibrium steady state) probability vector is

$$
\langle\mathbf{P}\rangle_{\xi}=\lim _{s \rightarrow 0} s\langle\widetilde{\mathbf{P}}(s)\rangle_{\xi} .
$$

Substituting matrices W (27) and A (34) into the expression for the probability vector in the Laplace space (26), then multiplying it by $s$ and letting $s$ tend to zero, we obtain the steady state probability vector

$$
\begin{aligned}
\langle\mathbf{P}\rangle_{\xi}= & \frac{1}{4 \Gamma(\Gamma+\alpha)-\Gamma_{L}^{2} \gamma^{2}} \\
& \times\left[\begin{array}{c}
2(\Gamma+\alpha)\left\{\Gamma_{L}\left(1-f_{L}\right)+\Gamma_{R}\left(1-f_{R}\right)\right\}-\Gamma_{L}^{2} \gamma^{2}\left(1-f_{L}\right) \\
2(\Gamma+\alpha)\left\{\Gamma_{L} f_{L}+\Gamma_{R} f_{R}\right\}-\Gamma_{L}^{2} \gamma^{2} f_{L}
\end{array}\right],
\end{aligned}
$$

where we introduced $\Gamma=\left(\Gamma_{L}+\Gamma_{R}\right) / 2$. Notice that any dependence on initial probability vector $\mathbf{P}(0)$ completely disappears from the expression in the steady state. In the noiseless limit $(\gamma=0)$, we get

$$
\langle\mathbf{P}\rangle_{\xi}=\frac{1}{2 \Gamma}\left[\begin{array}{c}
\Gamma_{L}\left(1-f_{L}\right)+\Gamma_{R}\left(1-f_{R}\right) \\
\Gamma_{L} f_{L}+\Gamma_{R} f_{R}
\end{array}\right],
$$

which is the correct standard expression for the occupation probabilities of a single resonant-level.

The current is computed with the use of the continuity equation. The average number of electrons in the system is

$$
\langle N(t)\rangle_{\xi}=\left\langle P_{1}(t)\right\rangle_{\xi} .
$$

The continuity equation

$$
\frac{d}{d t}\left\langle P_{1}(t)\right\rangle_{\xi}=J_{L}(t)+J_{R}(t)
$$

is used to identify expressions for the electron current

$$
\langle J\rangle_{\xi}=\left\langle J_{L}\right\rangle_{\xi}=-\left\langle J_{R}\right\rangle_{\xi}=\Gamma_{R}\left[-f_{R},\left(1-f_{R}\right)\right] \cdot\langle\mathbf{P}\rangle_{\xi}
$$

Computing the dot product in (40), we get

$$
\langle J\rangle_{\xi}=\frac{\Gamma_{L} \Gamma_{R}\left(2 \Gamma+2 \alpha-\Gamma_{L} \gamma^{2}\right)}{4 \Gamma(\Gamma+\alpha)-\Gamma_{L}^{2} \gamma^{2}}\left(f_{L}-f_{R}\right) .
$$

Figures 1 and 2 show the current-voltage characteristics of a single resonant-level averaged over realisations of the

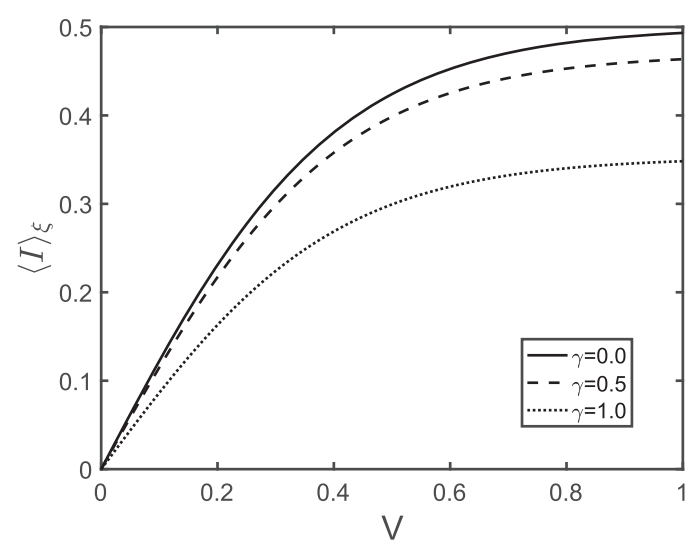

FIG. 1. Noise averaged current for a single resonant-level as a function of the applied voltage bias for different values of the amplitude of the telegraph noise $\gamma$. Parameters used in calculations: $\Gamma_{R}=\Gamma_{L}=\Gamma=1, T=0.1$, and $\epsilon=0$-all energy units are given in terms of $\Gamma$. The unit for electric current is $\Gamma$ (or if we put $\hbar$ and $e$ back, it is $e \Gamma / \hbar$ ) and values of voltage bias $V_{s d}$ are given in $\Gamma$.

stochastic telegraph process. The noise in molecular electrode contact reduces the electric current and also reduces the molecular conductivity (the slope of the I-V curve). The reduction of the current is more significant for the larger amplitudes of the telegraph noise $\gamma$. The rare telegraph processes (the small density of telegraph jump points on the time axis, $\alpha$ ) give the larger reduction and very frequent jumps bring the current to the noiseless limit. To get physical insight, we may consider the ratio $\alpha / \Gamma$ as the number of telegraph jumps per average time required for an electron to tunnel across the molecule. Therefore, with our choice of the parameters, $\alpha=5$ roughly corresponds to 5 telegraph switching per one tunneling electron and $\alpha=0.1$ represents one telegraph jump during approximately 10 electron tunneling events.

\section{B. Telegraph noise in molecular junctions with electron-vibration coupling}

Let us consider the case of the electron transport through a molecular junction with electron-vibration interaction. The molecular bridge itself is described by Holstein Hamiltonian

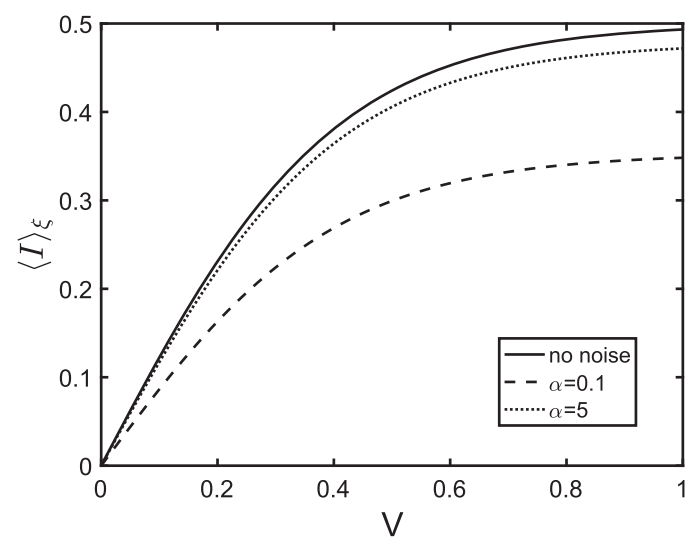

FIG. 2. Noise averaged current for a single resonant-level as a function of the applied voltage bias for different values of the number of telegraph jumps per unit time $\alpha$. Parameters and units are the same as in Fig. 1. 


$$
H_{\text {molecule }}=\epsilon_{0} a^{\dagger} a+\lambda \omega\left(b^{\dagger}+b\right) a^{\dagger} a+\omega b^{\dagger} b,
$$

where $\epsilon_{0}$ is the molecular orbital energy, $\omega$ is the molecular vibration energy, and $\lambda$ is the strength of the electron-vibration coupling. $a^{\dagger}(a)$ creates (annihilates) an electron on the molecular orbital and $b^{+}(b)$ is the bosonic creation (annihilation) operator for the molecular vibration.

We assume that the vibration maintains the equilibrium Bose-Einstein distribution at all time and, as a result, we obtain the thermally averaged rates for the electron transfers $(m, n=0,1)$

$$
T_{m n}^{L / R}=\sum_{q q^{\prime}} \Gamma_{m q, n q^{\prime}}^{L / R} \frac{e^{-q^{\prime} \omega / T}}{1-e^{-\omega / T}} .
$$

The individual transition rates between microscopic molecular states are computed using the Fermi golden rule. ${ }^{40}$

$$
\Gamma_{0 q^{\prime}, 1 q}^{L / R}=\Gamma_{L / R}\left|X_{q^{\prime} q}\right|^{2}\left(1-f_{L / R}\left[\epsilon-\omega\left(q^{\prime}-q\right)\right]\right)
$$

is the rate for the transition from the state occupied by one electron and $q$ vibrations to the electronically unoccupied state with $q^{\prime}$ vibrations by the electron transfer from the molecule to left and right electrodes, respectively, and

$$
\Gamma_{1 q^{\prime}, 0 q}^{L / R}=\Gamma_{L / R}\left|X_{q^{\prime} q}\right|^{2} f_{L / R}\left[\epsilon+\omega\left(q^{\prime}-q\right)\right]
$$

is the rate for the transition when electron is transferred from the left/right electrode into the originally empty molecules simultaneously changing the vibrational state from $q$ to $q^{\prime}$. These rates depend on the electronic level broadening functions $\Gamma^{L / R}$, the Fermi occupation numbers $f_{L / R}$, and the Franck-Condon factors $X_{q q^{\prime}}$.

The matrices $\mathbf{W}$ and $\mathbf{A}$ have the same structure as in the transport through a single resonant-level that are (27) and (34) with the transitional rates defined by (43). Likewise to the electron transport through a single resonant-level, we compute steady state probabilities and electric current which are averaged over the realisations of the telegraph process.

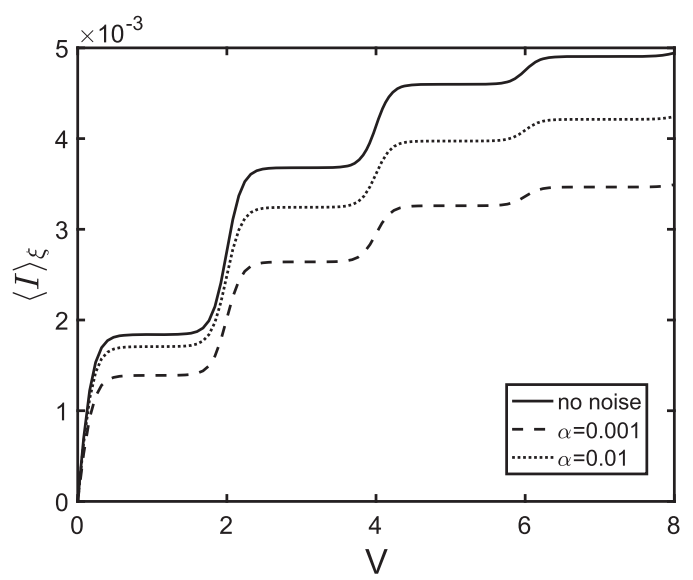

FIG. 3. Noise averaged current in a molecular junction with electronvibration interaction as a function of the applied voltage bias computed for different values of the number of telegraph jumps per unit time. Parameters used in the calculations: $\Gamma_{R}=0.01, \Gamma_{L}=0.01, T=0.05, \lambda=1, \omega=1$, $\epsilon=0$-all energy units are measured in terms of vibrational frequency energy $\omega$. The unit for electric current is $\omega$ (or if we put $\hbar$ and $e$ back, it is $e \omega / \hbar$ ) and values of voltage bias $V_{s d}$ are given in $\omega$.
Figure 3 shows the current as a function of the applied voltage computed for different densities of telegraph jump points on the time axis. The steps in the current correspond to the resonant excitations of the vibration states by electric current which occur when the voltage passes through an integer multiple of the vibration energy. ${ }^{40-42}$ As in the single resonant-level case considered before, the telegraph noise in the molecule-electrode contacts results in the reduction of the electric current, but the frequent switching events (large $\alpha$ ) again bring the I-V curve to the optimal noiseless limit.

\section{CONCLUSIONS}

We have developed a theoretical approach to solve Markovian master equations with a telegraph noise. The rates of the master equation depend on a set of time-dependent parameters, which switch randomly between two values. We treated the probabilities as functionals of a stochastic telegraph process. We used Novikov's method, originally developed in the theory of turbulence, to shift the stochastic functionals to the deterministic function domain; this procedure converted the stochastic master equation to a closed system of deterministic differential equations. The equations were solved with the use of the Laplace transformation, and the general expression for the probability vector averaged over realisations of the stochastic process was derived. The theory was applied to quantum electron transport through model molecular junctions. We studied the role of the noise in the molecule-electrode contacts on the current-voltage characteristics. Two models were considered: a single resonant-level transport and the Holstein model in the polaronic regime. In both models, the contact telegraph noise reduces the electric current and lowers the molecular conductivity.

${ }^{1}$ R. Avriller and A. Levy Yeyati, Phys. Rev. B 80, 041309 (2009).

${ }^{2}$ C. Schinabeck, R. Härtle, H. B. Weber, and M. Thoss, Phys. Rev. B 90, 075409 (2014).

${ }^{3}$ B. K. Agarwalla, J.-H. Jiang, and D. Segal, Phys. Rev. B 92, 245418 (2015).

${ }^{4}$ O. Tal, M. Krieger, B. Leerink, and J. M. van Ruitenbeek, Phys. Rev. Lett. 100, 196804 (2008).

${ }^{5}$ S. L. Rudge and D. S. Kosov, J. Chem. Phys. 144, 124105 (2016).

${ }^{6}$ D. S. Kosov, J. Chem. Phys. 146, 074102 (2017).

${ }^{7}$ D. S. Kosov, J. Chem. Phys. 147, 104109 (2017).

${ }^{8}$ N. Néel, J. Kröger, and R. Berndt, Nano Lett. 11, 3593 (2011).

${ }^{9}$ D. Djukic and J. M. van Ruitenbeek, Nano Lett. 6, 789 (2006).

${ }^{10}$ M. Tsutsui, M. Taniguchi, and T. Kawai, Nat. Commun. 1, 138 (2010).

${ }^{11}$ Y.-C. Chen and M. Di Ventra, Phys. Rev. Lett. 95, 166802 (2005).

${ }^{12}$ M. Galperin, A. Nitzan, and M. A. Ratner, Phys. Rev. B 74, 075326 (2006).

${ }^{13}$ M. Kiguchi, O. Tal, S. Wohlthat, F. Pauly, M. Krieger, D. Djukic, J. C. Cuevas, and J. M. van Ruitenbeek, Phys. Rev. Lett. 101, 046801 (2008).

${ }^{14}$ F. Haupt, T. Novotný, and W. Belzig, Phys. Rev. Lett. 103, 136601 (2009).

${ }^{15}$ N. L. Schneider, J. T. Lü, M. Brandbyge, and R. Berndt, Phys. Rev. Lett. 109, 186601 (2012).

${ }^{16}$ Y. Utsumi, O. Entin-Wohlman, A. Ueda, and A. Aharony, Phys. Rev. B 87, 115407 (2013).

${ }^{17}$ Y. V. Nazarov and Y. M. Blanter, Quantum Transport: Introduction to Nanoscience (Cambridge University Press, 2009).

${ }^{18}$ Y. Kim, H. Song, D. Kim, T. Lee, and H. Jeong, ACS Nano 4, 4426 (2010).

${ }^{19}$ J. M. Artes, M. Lopez-Martinez, I. Diez-Perez, F. Sanz, and P. Gorostiza, Small 10, 2537 (2014).

${ }^{20}$ Y. Song, H. Jeong, S. Chung, G. H. Ahn, T.-Y. Kim, J. Jang, D. Yoo, H. Jeong, A. Javey, and T. Lee, Sci. Rep. 6, 33967 (2016). 
${ }^{21}$ D. Cho, H. Lee, S. Shekhar, M. Yang, J. Y. Park, and S. Hong, Sci. Rep. 7, 43411 (2017).

${ }^{22}$ R. Nichols, W. Haiss, S. J. Higgins, E. Leary, S. Martin, and D. Bethell, Phys. Chem. Chem. Phys. 12, 2801 (2010).

${ }^{23}$ Y. M. Galperin, N. Zou, and K. A. Chao, Phys. Rev. B 49, 13728 (1994).

${ }^{24}$ Y. M. Galperin and K. A. Chao, Phys. Rev. B 52, 12126 (1995).

${ }^{25}$ S. Gurvitz, A. Aharony, and O. Entin-Wohlman, Phys. Rev. B 94, 075437 (2016).

${ }^{26}$ O. Entin-Wohlman, D. Chowdhury, A. Aharony, and S. Dattagupta, Phys. Rev. B 96, 195435 (2017).

${ }^{27}$ E. A. Novikov, Sov. Phys. JETP 20, 1290 (1965).

${ }^{28}$ V. I. Klyatskin, Dynamics of Stochastic Systems (Elsevier, Amsterdam, 2005).

${ }^{29}$ V. I. Klyatskin, Stochastic Equations Through the Eye of the Physicist: Basic Concepts, Exact Results and Asymptotic Approximations (Elsevier, Amsterdam, 2005).
${ }^{30}$ V. I. Klyatskin, Lectures on Dynamics of Stochastic Systems (Elsiever, Amsterdam, 2011).

${ }^{31}$ J. Keilson and J. E. Storer, Q. Appl. Math. 10, 243 (1952).

${ }^{32} \mathrm{~S}$. Temkin and A. I. Burshtein, Spectroscopy of Molecular Rotation in Gases and Liquids (Cambridge University Press, Cambridge, 1994).

${ }^{33}$ A. Blokhin and M. Gelin, Mol. Phys. 87, 455 (1996).

${ }^{34}$ M. F. Gelin, Chem. Phys. 240, 265 (1999).

${ }^{35}$ M. F. Gelin and D. S. Kosov, J. Chem. Phys. 124, 144514 (2006).

${ }^{36}$ M. F. Gelin, A. P. Blokhin, V. A. Tolkachev, and W. Domcke, Chem. Phys. 462, 35 (2015).

${ }^{37}$ A. I. Shushin, J. Chem. Phys. 114, 4883 (2001).

${ }^{38}$ N. G. Van Kampen, Stochastic Processes in Physics and Chemistry, 3rd ed. (North-Holland Personal Library, Amsterdam, 2007).

${ }^{39}$ V. Shapiro and V. Loginov, Phys. A 91, 563 (1978).

${ }^{40}$ A. Mitra, I. Aleiner, and A. J. Millis, Phys. Rev. B 69, 245302 (2004).

${ }^{41}$ R. Härtle and M. Thoss, Phys. Rev. B 83, 115414 (2011).

${ }^{42}$ J. Koch and F. von Oppen, Phys. Rev. Lett. 94, 206804 (2005). 